\title{
Fieber unklarer Genese (FUO) bei Kindern und Jugendlichen mit onkologischen Erkrankungen
}

Diagnostic Procedures and Management of Fever in Pediatric Cancer Patients

\section{Zusammenfassung}

Die Einführung einer unverzüglich begonnenen empirischen intravenösen Antibiotikatherapie hat die Letalität von onkologisch erkrankten Patienten mit Fieber während der Granulozytopenie deutlich gesenkt. So ist bei Sepsis mit Gramnegativen Erregern die Letalität von $84 \%$ in den 1960 er-Jahren auf 3-10\% in aktuellen Untersuchungen reduziert worden. Allerdings erlaubt die initiale klinische Präsentation des Patienten nur selten eine eindeutige Einschätzung hinsichtlich der Schwere des weiteren klinischen Verlaufes. Auch der weitere Verlauf führt nur in etwa der Hälfte der Patienten zu einer klinischen oder mikrobiologischen Diagnose, so dass etwa 50\% der Patienten mit Fieber unbekannter Ursache (FUO) behandelt werden. Während für die Behandlung internistisch onkologischer Patienten Leitlinien zur Therapie des FUO auf der Grundlage von Studien existieren, ist die Datenlage für onkologisch erkrankte Kinder und Jugendliche deutlich schlechter. Die Einleitung der empirischen Therapie mit einem Pseudomonas-wirksamem Antibiotikum wird allgemein anerkannt. Wenig umstritten ist auch die stationäre Aufnahme der Patienten und der sofortige Beginn der intravenösen antibiotischen Therapie, ohne den Erregernachweis abzuwarten. Unklarheiten bestehen jedoch hinsichtlich der Indikation einer Therapie mit Aminoglykosiden und Glykopeptiden. Auch Therapiedauer und die Notwendigkeit der Antibiotika-Ergänzung und -Umstellung werden uneinheitlich gehandhabt. Auf der Grundlage von Studien an onkologisch erkrankten Erwachsenen und soweit verfügbar auch von Kindern und Jugendlichen, werden die oben genannten offenen Fragen dargestellt und diskutiert. Die daraus resultierenden Empfehlungen werden für Kinder

\section{Abstract}

The rapid institution of empirical broad-spectrum antibiotics has become the gold standard of treatment for febrile neutropenic children undergoing therapy for cancer. With this approach, morbidity and mortality have dropped significantly but have not been eliminated altogether. In recent randomized studies evaluating different drug combinations, there is still a $3-10 \%$ mortality reported in febrile, neutropenic cancer events. Despite improvements in invasive and non-invasive diagnostic procedures, a majority of patients will receive antibiotic therapy despite an inability to identify a specific pathogen or source (fever of unknown origin, FUO). While there are evidence-based guidelines in adult patients with fever and neutropenia, data are less clear in the pediatric population. Experts agree on the early use of empirical antibiotic therapy, which covers Pseudomonas spp. and is initiated at the first sign of fever. The success of this approach has been based upon clinical intervention before the results of the diagnostic evaluation are available. In contrast, the use of aminoglycosides or glycopeptides is still a matter of debate, as it is the duration of antibiotic therapy. Based on published data in pediatric and adult patients with cancer, the current diagnostic procedures and therapeutic strategies will are discussed. The recommendations given are a consensus of the German Society of Pediatric Oncology and Hematology (GPOH) and the German Society of Pediatric Infectious Diseases (DGPI). 
und Jugendliche angepasst. Dabei sind lokale Erregerspektren einzubeziehen.

\section{Schlüisselwörter}

Fieber unklarer Ursache · FUO · Kinderonkologie · Empfehlung

\section{Key words}

Fever of unknown origin · FUO · oncology · children · recommondation
Die Prognose von Kindern und Jugendlichen mit einer malignen Erkrankung hat sich in den letzten Jahrzehnten deutlich verbessert [10]. Dieser Erfolg ist hauptsächlich auf die Entwicklung risikoadaptierter Therapieprotokolle zurückzuführen, in denen eine optimierte chemotherapeutische, radiotherapeutische und/oder chirurgische Behandlung aufeinander abgestimmt wird. Als weiterer Meilenstein gilt die Verbesserung der supportiven Therapie, sowohl in der Prophylaxe als auch in der Therapie infektiöser Komplikationen. So ist es zum Beispiel aufgrund des Einsatzes der empirischen Antibiotikatherapie gelungen, die Letalität bei bakteriellen Infektionen und Sepsis von $84 \%$ in den 1960er-Jahren auf $44 \%$ in den 1970 er-Jahren [6] und weiter auf $3-10 \%$ in aktuellen Untersuchungen zu reduzieren [3, 27, 40, 64]. Grundlegende Informationen zu Risikofaktoren und Erregerspektrum sind im einleitenden Artikel (Lehrnbecher und Laws) aufgezeigt. Fieber während der Granulozytopenie ohne Erregernachweis und erkennbarer Infektionsursache wird als Fieber unbekannter Ursache (FUO) bezeichnet und macht etwa 50-60\% der Infektionen in der Onkologie aus [42]. Entsprechend den derzeitigen Empfehlungen werden Kinder mit Granulozytopenie und Fieber stationär aufgenommen und sofort mit einer empirischen intravenösen antibiotischen Therapie behandelt, ohne den Erregernachweis abzuwarten. In diesem Kapitel sollen hierzu die diagnostischen und therapeutischen Überlegungen dargestellt werden. Sie berücksichtigen die Leitlinien der Paul-Ehrlich-Gesellschaft, der Infectious Diseases Society of America und der Arbeitsgemeinschaft Infektionen in der Hämatologie und Onkologie $[29,43]$. Limitierend sind dabei oftmals fehlende, aber dringend notwendige Studien zur Kinetik und Arzneimittelsicherheit bei Kindern und Jugendlichen [25].

\section{Definitionen}

Fieber und Granulozytopenie werden sowohl in der Literatur als auch im klinischen Alltag unterschiedlich definiert. Es wäre wünschenswert, wenn die vorgeschlagenen Definitionen zukünftig einheitlich verwendet würden, um auch die Vergleichbarkeit von Studien zu vereinfachen.

\section{Granulozytopenie}

Neutrophile Granulozyten (neutrophile Granulozyten: Segmentund Stabkernige $<500 / \mu l\left(0,5 \times 10^{9} / 1\right)$ oder $<1000 / \mu l$ mit $\mathrm{zu}$

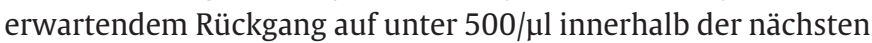
zwei Tage.

\section{Fieber}

Oral, axillär oder in der Kinderonkologie seltener rektal gemessene Temperatur

- einmalig $\geq 38,5^{\circ} \mathrm{C}$ oder

- zweimalig $\geq 38^{\circ} \mathrm{C}$ für eine Stunde anhaltend innerhalb von $12 \mathrm{~h}$.

\section{Unerklärtes Fieber}

Als unerklärtes Fieber oder Fieber unbekannter Genese („fever of unknown origin“, FUO) wird neu aufgetretenes Fieber ohne richtungsweisende klinische oder mikrobiologische Infektionsbefunde gewertet [12].

\section{Klinisch gesicherte Infektion}

Eine klinisch gesicherte Infektion („clinically documented/defined infection“, CDI) ist ein diagnostisch eindeutig lokalisierter Befund, beispielsweise einer Pneumonie, die klinisch oder radiologisch nachgewiesen ist, oder einer Haut-Bindegewebe-Infektion, deren mikrobiologische Pathogenese jedoch nicht bewiesen werden kann oder die einer Untersuchung nicht zugänglich ist.

\section{Mikrobiologisch gesicherte Infektion mit oder ohne Bakteriämie}

Eine mikrobiologisch gesicherte Infektion („microbiologically documented/defined infection“, MDI) liegt vor, wenn neben einem lokalisierbaren Infektionsbefund ein zeitlich und mikrobiologisch plausibler Erregernachweis gelingt oder wenn Infektionserreger in der Blutkultur auch ohne lokalisierten Infektionsherd nachweisbar sind (Bakteriämie). Details sind den Artikeln von Groll et al. und Simon et al. in diesem Band zu entnehmen.

\section{Diagnostik}

Fieber stellt häufig das einzige Symptom einer Infektion oder Sepsis eines granulozytopenischen Patienten dar. Daher sind Patienten mit Fieber bei Granulozytopenie unverzüglich und gründlich zu untersuchen (Tab. 1). Die körperliche Untersuchung ist kurzfristig zu wiederholen. Selbstverständlich gilt dies auch für nicht fiebernde onkologische Patienten, die sich im reduzierten Allgemeinzustand oder mit neu aufgetretenen infektionsverdächtigen klinischen Symptomen vorstellen.

Vor dem Beginn einer empirischen Antibiotikatherapie sind Laborparameter zu bestimmen sowie Blutkulturen aus allen Lumen eines Katheters zu entnehmen (siehe Kapitel Beutel und Simon). Aufgrund fehlender klinischer Konsequenzen verzichten die meisten Kinderonkologen auf eine zusätzliche Blutkultur aus einer peripheren Vene.

Die weitere mikrobiologische Diagnostik erfolgt gezielt, d.h. Abstriche, Liquorpunktionen sowie Urin- und Stuhlkulturen sind nur bei entsprechender Symptomatik oder Fragestellung indiziert.

Eine erweiterte mikrobiologische Diagnostik ohne bestehende Symptome hat keine Auswirkung auf die einzuleitende empirische Behandlung [23]. 
Tab. 1 Initiale Diagnostik bei FUO

\begin{tabular}{|c|c|}
\hline $\begin{array}{l}\text { körperliche } \\
\text { Untersuchung }\end{array}$ & $\begin{array}{l}\text { Vollständige Untersuchung einschließlich } \\
\text { - Prädilektionsstellen: } \\
\text { - Haut- und Schleimhaut } \\
\text { - Perianalregion } \\
\text { - Kathetereintritt } \\
\text { - Nagelfalz } \\
\text { - Blutdruck und Puls } \\
\text { - Atemfrequenz } \\
\text { - Schmerzsymptomatik } \\
\text { - Körpertemperatur }\end{array}$ \\
\hline Laborparameter & $\begin{array}{l}\text { Blutbild mit Differenzialblutbild } \\
\text { C-reaktives Protein } \\
\text { Blutzucker } \\
\text { Transaminasen, Bilirubin } \\
\text { Kreatinin, } \\
\text { Natrium, Kalium } \\
\text { PTT, Quick } \\
\text { bei Oberbauchschmerzen } \\
\text { - Lipase } \\
\text { bei Sepsis-Verdacht } \\
\text { - Blutgasanalyse } \\
\text { - Laktat } \\
\text { - ATIII }\end{array}$ \\
\hline $\begin{array}{l}\text { mikrobiologische } \\
\text { Untersuchung }\end{array}$ & $\begin{array}{l}\text { Blutkulturen aus allen Lumen eines venösen Zuganges } \\
\text { entsprechend Klinik } \\
\text { - Wund- oder Körperabstriche, Liquor, Urin, Stuhl }\end{array}$ \\
\hline Bildgebung & $\begin{array}{l}\text { nur bei klinischen Hinweisen } \\
\text { - Röntgen-Thorax } \\
\text { - Sonographie-Abdomen } \\
\text { - CT Lunge, CT NNH }\end{array}$ \\
\hline
\end{tabular}

Bezüglich der bildgebenden Diagnostik kann bei Kindern und Jugendlichen bei fehlenden klinischen Hinweisen auf ein initiales Röntgenbild der Lunge verzichtet werden [32, 37, 56]. Falls bei klinischer Symptomatik wie Dyspnö ein Röntgenbild durchgeführt wird, ist ein a.p.-Röntgenbild der Lunge im Sinne einer möglichst geringen Strahlenbelastung ausreichend. Dabei bleibt jedoch das Risiko bestehen, eine kleinere basale Pneumonie zu übersehen. Ebenfalls kann bei fehlender Klinik initial auf eine Sonographie des Abdomens verzichtet werden.

\section{Initiale empirische Therapie}

Die Indikation zu einer kalkulierten empirischen Therapie besteht für alle Kinder und Jugendlichen mit Fieber während der Granulozytopenie. Unabhängig von der Granulozytenzahl werden Kinder und Jugendliche mit Krebserkrankungen empirisch mit Antibiotika behandelt, wenn sie Hinweise auf eine Sepsis oder auf eine klinisch dokumentierte Infektion bieten. Nach klinischer Diagnosestellung ist die Therapie unverzüglich zu beginnen. Eine abwartende Haltung bis zum Eintreffen mikrobiologischer Ergebnisse ist nicht gerechtfertigt, da die Letalität einer unbehandelten Infektion bis zu 50\% innerhalb von 48 Stunden reicht [14].

Die empirische antibakterielle Therapie muss bei Kindern mit Krebserkrankungen ein breites Erregerspektrum erfassen, das insbesondere auch Gramnegative Erreger wie Pseudomonas aeruginosa und Klebsiella spp. beinhaltet, da Infektionen mit diesen Erregern oft fulminante und dann therapeutisch nicht mehr beherrschbare Verläufe zeigen [60].
Auf der anderen Seite soll eine antibakterielle Therapie gleichzeitig nur eine geringe Toxizität aufweisen. Da im Gegensatz zu erwachsenen Patienten mit Granulozytopenie und Fieber eine orale empirische Therapie außerhalb von Studien nicht empfohlen wird, ist die antibakterielle Substanz intravenös zu applizieren.

Bei der Auswahl der antimikrobiellen Substanzen sind sowohl lokales bzw. regionales Erregerspektrum als auch die Resistenzsituation zu berücksichtigen, wie sie von der Paul-Ehrlich-Gesellschaft für Chemotherapie e.V. veröffentlicht werden [38]. Außerdem müssen spezielle Patientencharakteristika (z.B. Komorbidität) und das jeweilige zytotoxische Behandlunsregime in die Überlegungen mit einbezogen werden. So haben zum Beispiel Patienten, die mit hochdosiertem Cytarabin behandelt wurden, ein hohes Risiko, eine Infektion durch Streptokokken der Viridans-Gruppe zu erleiden. Diese Infektionen sind im Gegensatz zu Infektionen mit Koagulase-negativen Staphylokokken oder Corynebakterien oft sehr schwer verlaufend und haben eine hohe Letalität [40].

Da die Mehrzahl der Patienten mit den verfügbaren kalkulierten antibiotischen Initialtherapien innerhalb von 72 Stunden entfiebert $[8,24]$, ist die Fallzahl der meisten klinischen Studien zu klein, um eine Überlegenheit eines Medikamentes oder einer Medikamentenkombination zu belegen. Zudem sind viele Studien schwer vergleichbar, da sie unterschiedliche Patientenkollektive einschließen oder unterschiedliche Endpunkte analysieren.

Insgesamt können verschiedene Antibiotika unterschiedlicher Substanzklassen für eine Monotherapie bzw. als Bestandteil einer Kombinationstherapie empfohlen werden. Vorteile einer Monotherapie sind geringere Infusionsmengen und eine geringere Infusionsdauer, was gerade bei pädiatrischen Patienten eine besondere Rolle spielen kann. Zudem weist eine Monotherapie oft eine geringere Toxizität und ein günstigeres Interaktionspotenzial auf. Auf der anderen Seite sprechen ein Synergismus von antibakteriellen Medikamenten und eine geringere Gefahr der Resistenzbildung für eine Kombinationstherapie.

Die oben genannten Kriterien werden derzeit von Cephalosporinen der 3. oder 4. Generation (Ceftazidim und Cefepim) sowie von Carbapenemen (Imipenem-Cilastat oder Meropenem) erfüllt, zu denen es auch pädiatrische Studien gibt $[7,18,30$, 33, 39]. Diese Medikamente haben nur eine geringe Wirksamkeit auf Koagulase-negative Staphylokokken und keine Wirksamkeit auf Methicillin-resistente Staph. aureus. Lediglich Cefepim und die Carbapeneme weisen eine gute Aktivität gegen Streptokokken der Viridans-Gruppe und Pneumokokken auf. Ein anderer Nachteil des Ceftazidim ist die potenzielle Resistenz einiger Gramnegativer Bakterien durch Extended-Spektrum Beta-Laktamasen oder Typ-1-Beta-Laktamasen.

In der internistischen Onkologie ist die fixe Kombination von Piperacillin mit Tazobactam als Monotherapie evaluiert und wird bei FUO empfohlen $[8,43]$. Piperacillin/Tazobactam weist eine gute Verträglichkeit auf und hat ein breites antimikrobiologisches Wirkungsspektrum. Auch wenn diese fixe Kombination bereits ab dem Neugeborenenalter verwendet wird [5, 20,41] 
ist zu berücksichtigen, dass es nur wenige klinische Studien bei onkologisch erkrankten Kindern mit FUO hierzu gibt [8, 9, 19, 48] und dass sie erst ab dem zweiten Lebensjahr bei intraabdominellen Infektionen zugelassen ist. Für die freie Kombination aus Piperacillin/Sulbactam gibt es derzeit keine ausreichend gesicherten klinischen Studien in der Onkologie. Die Resistenztestung auf Piperacillin/Tazobactam ist nicht auf Piperacillin/Sulbactam übertragbar. Außerdem sind aktuelle Änderungen der DIN-Grenzwerte für Beta-Lactamase-Inhibitoren zu berücksichtigen.

Obwohl die Carbapeneme gut vertragen werden und effektiv sind [18], sollten sie aus Kostengründen und zur Vermeidung von Resistenzen der Therapieintensivierung vorbehalten bleiben. Weitere Details im Artikel von Simon A et al.

Für eine Kombinationstherapie gibt es im Wesentlichen die Möglichkeit, die oben genannten Medikamente - Ceftazidim, Cefepim, Imipenem-Cilastat oder Meropenem, PiperacillinTazobactam - sowie Ceftriaxon mit einem Aminoglycosid wie Gentamycin, Tobramycin oder Amikacin zu kombinieren. Betalaktam-Antibiotika und Aminoglykoside zeigen einen synergistischen Effekt auf Gramnegative Erreger und Staphylokokken, weshalb diese Kombination gerade für schwer kranke Kinder bevorzugt wird. Allerdings ist der mögliche Vorteil einer Kombination mit Aminoglykosiden den Nebenwirkungen wie Ototoxizität oder Nephrotoxizität gegenüberzustellen. Für die Kombinationstherapie gibt es zahlreiche pädiatrische Studien, die Wirksamkeit und Verträglichkeit belegen [2] Anzumerken bleibt aber, dass Kombinationstherapien mit Aminoglykosiden meistens gegen die Monotherapie mit Carbapenemen geprüft wurden und nur selten gegen das für die Kombination gewählte Ureidopenicillin oder Cephalosporin [2]. In zwei kürzlich publizierten Metaanalysen bei Patienten mit Fieber bei Granulozytopenie ist gezeigt worden, dass die Kombinationstherapie mit einem Aminoglykosid keine Vorteile gegenüber einer Monotherapie erbringt $[22,53]$. Auch bei der Behandlung immunkompetenter Patienten mit Sepsis hat die zusätzliche Gabe eines Aminoglykosides nicht die Mortalität vermindert, führte aber zu mehr Nebenwirkungen [52].

Empfehlung: Unter Berücksichtigung des lokalen Erregerspektrums und dessen Resistenzlage, sowie der individuellen Patientenanamnese soll ein Beta-Lactam Antibiotikum mit Pseudomonasaktivität verwendet werden. Aminoglykoside sind bei der empirischen Initialtherapie in aller Regel verzichtbar.

Einsatz von Glykopeptiden in der empirischen Initialtherapie Der generelle Einsatz von Glycopeptiden (Vancomycin, Teicoplanin) in der empirischen Initialtherapie erscheint nicht gerechtfertigt $[11,17,29]$. Zum einen birgt der breite Einsatz dieser Medikamente die Gefahr von Resistenzen, insbesondere von Vancomycin-resistenten Enterokokken [1]. Zum anderen werden praktisch alle Erreger von fulminant verlaufenden Infektionen durch die oben genannten Medikamente der kalkulierten Initialtherapie erfasst $[8,55]$. Aus diesem Grund ist der Einsatz von Glycopeptiden nach Erhalt der mikrobiologischen Ergebnisse in aller Regel ausreichend, ohne die Patienten zu gefährden $[15,36]$.
Eine Ausnahme stellen lediglich Kliniken dar, in denen sehr häufig schwere Infektionen durch Methicillin-resistente Pneumokokken oder Staph. aureus und Streptococcus viridans gesehen werden, da diese in der Hälfte der Fälle resistent gegen Penicillin sind [61]. Zusätzlich kann eine empirische Initialtherapie mit Glykopeptiden bei Patienten nach hochdosierter Cytarabin-Gabe oder bei Patienten, bei denen eine schwere Katheter-assoziierte Infektion vermutet wird, erwogen werden [29], insbesondere, wenn in der Institution häufig Penicillin-resistente vergrünende Streptokokken gefunden werden. Bei Patienten mit kardiovaskularer Insuffizienz wie Hypotension oder verzögerter Rekapillisationszeit, ist der empirische Einsatz eines Gykopeptides sinnvoll.

Empfehlung: Glykopeptide sind primär nur bei Katheter-assoziierten Infektionen oder in Abteilungen mit hoher MRSAPrävalenz verwenden. Der Einsatz von Glycopeptiden in der Initialtherapie bei Patienten nach hochdosiertem Cytarabin kann erwogen werden. Bei kardiovaskulärer Insuffizienz des Patienten ist der Einsatz sinnvoll.

\section{FUO und abdominelle oder perianale Infektionszeichen}

Bei entsprechender Klinik sollen Antibiotika mit guter Wirksamkeit gegen Anaerobier verwendet werden. Bei Carbapenemen bzw Piperacillin/Tazobactam ist keine Ergänzung notwendig. Bei Cephalosporinen mit Wirksamkeit gegen Pseudomonaden ist die Ergänzung mit Metronidazol sinnvoll. Dabei ist die orale Gabe zur Behandlung einer Clostridium difficile Enterokolitis wirkungsvoller als die intravenöse Applikation (siehe Kapitel Ballauf und Eggert).

\section{Therapiemodifikationen}

Das klinische Ansprechen auf eine gewählte empirische Antibiotikatherapie wird im Median erst nach 5-7 Tagen erreicht [13]. Deshalb ist bei klinisch stabilen Allgemeinzustand des Patienten eine Therapiemodifikation frühestens nach $72-96$ h in Erwägung zu ziehen, bei klinisch instabilen Patienten jedoch eher. Bevor die Therapiemodifikation durchgeführt wird, sind folgende Aspekte zu bedenken:

- Liegt evtl. eine nicht-bakterielle Infektion vor?

- Liegt eine Zweitinfektion vor?

- Ist die Dosierung ausreichend?

- Werden im Zielorgan ausreichende Gewebespiegel erreicht?

- Kann ein Medikamentenfieber vorliegen?

- Liegt ein Abszess vor?

Vor dem Einleiten der Therapiemodifikation ist die Diagnostik gemäß Tab. 1 nochmals zu wiederholen und gegebenenfalls auszuweiten. Bei fehlenden Hinweisen auf eine dokumentierte Infektion bestehen in Abhängigkeit vom klinischen Zustand des Patienten sowie von der erwarteten Dauer der Granulozytopenie folgende Therapieoptionen:

- Beibehalten der bisherigen Therapie.

- Bei Monotherapie Ergänzung eines Aminoglykosides oder Glykopeptides.

- Bei Duotherapie: Wechsel zu Carbapenemen.

- Ergänzung eines Antimykotikums. 
Als bildgebende Diagnostik sind bei einem Patienten mit entsprechender Symptomatik ein hochauflösendes CT der Lunge (Spiral-CT oder Dünnschicht-CT) anzustreben, eine Sonographie des Abdomens und eine bildgebende Untersuchung der Nasennebenhöhlen durchzuführen.

Letztlich sollten durch die Therapiemodifikation mikrobiologische Lücken geschlossen werden. Zu diesem Zeitpunkt kann der Einsatz eines Glykopeptides erwogen werden. Bei fehlendem Nachweis von Grampositiven Erregern wurde in zwei Studien bei internistischen onkologischen Patienten gezeigt, dass kein Unterschied zwischen der monotherapeutischen Fortführung der Therapie mit Imipenem oder Piperacillin-Tazobactam und der Ergänzung mit Teicoplanin besteht $[8,16]$. Hingegen führte die Ergänzung eines Antimykotikums nach 72-96 h zu einer signifikant schnelleren Entfieberung, auch wenn keine Pilze nachgewiesen wurden [44, 62]. Beim Nachweis von Lungeninfiltraten im Röntgen- oder CT-Thorax ist dabei Amphothericin B dem Fluconazol überlegen [45, 59]. Der Stellenwert von neuen Antimykotika zur empirischen Therapie bei FUO ist derzeit nicht zu beurteilen [26].

Empfehlung: Bei initialer Verwendung von Carbapenemen oder Piperacillin-Tazobactam kann bei FUO über 72-96 h auf die Ergänzung mit einem Glykopeptid verzichtet werden (Ausnahme s. o.). Hingegen ist die Ergänzung eines Antimykotikums sinnvoll, das bei Nachweis von Lungeninfiltraten gegen Aspergillen wirksam ist.

\section{Dauer der Therapie}

Entsprechend den Leitlinien der Infectious Diseases Society of America wird bei FUO nach Entfieberung und beginnender

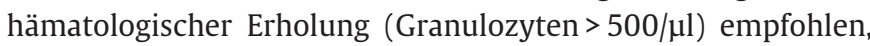
dass bei klinisch stabilen Patienten und unauffälligen Blutkulturen (sofern durchgeführt auch unauffälliger Rö-Thorax), die intravenöse Therapie $48 \mathrm{~h}$ später auf eine orale Therapie umgestellt wird [29]. Während internistische Patienten in einer Studie mit Amoxicillin plus Clavulansäure und Ciprofloxacin behandelt wurden [21], erhielten Kinder in zwei anderen Studien Cefixim $[51,58]$. Kritisch anzumerken ist, dass die orale Fortführung der Therapie unter stationären Bedingungen stattfand, so dass die Ergebnisse nicht ohne weiteres auf die ambulante Weiterbehandlung übertragbar sind. Die Mindesttherapiedauer beträgt gemäß diesen Richtlinien 7 Tage.

In einigen Studien konnte für Kinder und Jugendliche mit FUO unabhängig von der Neutrophilenzahl gezeigt werden, das $24 \mathrm{~h}$ nach Entfieberung und einer mindestens 72-stündigen intravenösen Antibiotikatherapie, diese unabhängig von der hämatologischen Erholung abgesetzt werden konnte, ohne dass eine orale Therapie durchgeführt wurde [31, 39, 57]. In zwei weiteren Studien wurde bei pädiatrischen Patienten gezeigt, dass bei diesen $24 \mathrm{~h}$ nach Entfieberung und im stabilen klinischen Allgemeinzustand die intravenöse Antibiotikatherapie beendet werden konnte und eine frühe Entlassung möglich war [34, 63]. Klaassen et al. beschreibt dabei in einer doppelblind durchgeführten, Plazebo-kontrollierten Studie, dass die Fortführung der Therapie mit einem oralen Antibiotikum und einsetzender hämatologischen Erholung nicht notwendig ist [34].

Bei anhaltender Granulozytopenie und klinisch stabilem Patienten muss daher zwischen dem potenziellen Risiko einer Reinfektion nach Absetzen der Antibiotikatherapie und der Resistenzbildung bzw. Superinfektion mit Pilzen bei Fortführung einer Antibiotikatherapie abgewogen werden. Eine Fortführung eventuell in modifizierter Form empfiehlt sich bei schwerer Granulozytopenie $<100 / \mu l$, dem Verdacht der Tumorinfiltration des Knochenmarks, bei oraler bzw. gastrointestinaler Mukositis, instabilen Patienten, einer klinisch oder mikrobiologischen Infektion oder bekannten Risikofaktoren. Als mögliche Konsequenzen bieten sich demnach die folgenden Möglichkeiten an

- Fortführung der intravenösen Therapie

- Wechsel auf die orale Therapie

- Absetzen der Therapie.

Empfehlung: Die intravenöse Antibiotikatherapie soll mindestens 72 Stunden betragen. Der klinisch stabile Patient soll vor Entlassung für mehr als $24 \mathrm{~h}$ fieberfrei sein und eine hämatologische Rekonstitution erkennbar sein. Die Fortführung der Behandlung mit einer oralen Antibiotikatherapie ist nicht notwendig.

\section{Antivirale Therapie}

Eine Antivirale Therapie soll nur erfolgen, wenn es klinische Hinweise auf das Vorliegen einer viralen Erkrankung gibt. Der empirische Einsatz ist nach derzeitigen Kenntnisstand nicht indiziert.

\section{Orale Antibiotikatherapie}

Für erwachsene Patienten hat die Multinational Association for Supportive Care in Cancer (MASCC, www.mascc.org) einen klinischen Risikoindex erarbeitet, der Patienten mit Fieber bei Granulozytopenie und einem niedrigen Risiko für Komplikationen mit einer guten Sensitivität und Spezifität erfasst [35]. Dieser Index, der multizentrisch und multinational entwickelt und validiert wurde, ist jedoch nicht auf Kinder und Jugendliche mit Fieber bei Granulozytopenie übertragbar, da beispielsweise bereits ein Alter unter 60 Jahren für ein niedriges Risiko spricht.

In der pädiatrisch-onkologischen Literatur fehlt ein Konsens, ob die orale antibiotische Therapie, meist in einem ambulanten Rahmen, den neuen Goldstandard der Behandlung von Fieber bei Granulozytopenie mit niedrigem Risiko invasiver bakterieller Infektionen darstellt oder nicht. Mullen [46] postuliert, dass ambulant verabreichte orale Antibiotika bei sorgfältig ausgewählten pädiatrischen Krebspatienten sicher und effektiv sind. Nach Hughes et al. [29] (IDSA-Leitlinien) stehen heute noch zu wenig Daten zur Verfügung, um eine empirische orale Antibiotikatherapie bei Kindern mit Fieber und Granulozytopenie zu empfehlen.

In der Kinderonkologie existieren viele monozentrische, jedoch keine multizentrischen oder gar multinationalen Studien [3], in denen die innerhalb weniger Stunden verfügbare Informationen 
aus Klinik und Hilfsuntersuchungen daraufhin untersucht wurden, ob sie als Kriterien zur Vorhersage schwerer Infektionen bei Fieber unter Granulozytopenie geeignet sind. Die verschiedenen aus diesen Einzelstudien resultierenden Risiko-Scores und -Kriterien widersprechen sich teilweise [47, 50, 54]. Der kleinste gemeinsame Nenner, die Kombination von gutem Allgemeinzustand, sich bereits erholender Knochenmarkfunktion und die Abwesenheit einer Komorbidität, ist zur Definition einer Episode von Fieber bei Granulozytopenie mit niedrigem Risiko einer schweren Infektion ungenügend [3, 28, 49]. Für pädiatrisch-onkologische Patienten mit Fieber bei Granulozytopenie ist somit keine Methode international etabliert und ausreichend validiert, die das Risiko einer schweren Infektion zu Beginn der Episode vorhersagen kann.

\section{Schlussfolgerungen}

In der pädiatrischen Onkologie fehlt weiterhin eine international anerkannte und validierte Definition einer Episode von Fieber bei Granulozytopenie mit einem niedrigen Risiko einer schweren Infektion. Für die tägliche klinische Praxis kann zur Behandlung von Kindern und Jugendlichen mit Fieber bei Granulozytopenie nur die sofortige Hospitalisierung und intravenöse Anti-

Tab. 2 Vorgehen bei pädiatrisch onkologischen Patienten mit Fieber bei Granulozytopenie während der intensiven Chemotherapie

Patient mit Fieber in der Granulozytopenie ohne Sepsis

- Monotherapie mit Pseudomonas wirksamem BreitspektrumBeta-Lactam-Antibiotikum

$\begin{array}{ll}\text { Patient mit Fieber in Granulozyto- } & \text { Zusatzdiagnostik I } \\ \text { penie mit Sepsis } & \text { bei entsprechender Klinik oder } \\ \text { - Carbapenem plus Glykopeptid } & \text { auffälliger Basisdiagnostik: } \\ & \text { Stuhlkultur (einschl. Clost.-diff.- } \\ & \text { Toxin und Virologie) } \\ & \text { Liquorpunktion } \\ & \text { Wundabstriche } \\ & \text { MS-Urin } \\ & \text { Sono-Abdomen } \\ \text { Rö CT NNH } & \text { CT-Thorax }\end{array}$

Modifikation: Persistierendes Fieber 72-96 h nach erster Antibiotikagabe und fehlender Erregernachweis, Patient in stabilem Zustand

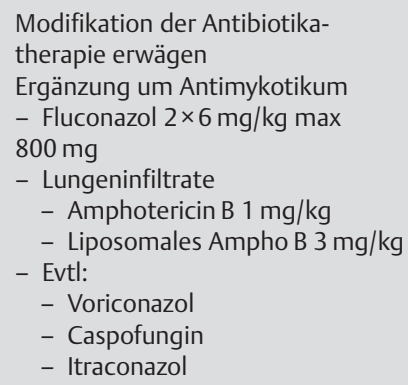

Therapiedauer: Intravenöse Antibiotikatherapie für mindestens $72 \mathrm{~h}$. Entlassung möglich bei klinisch stabilen Patienten, wenn mehr als $24 \mathrm{~h}$ fieberfrei und einsetzender hämatologischer Rekonstitution. Anmerkungen: Bei Unverträglichkeit Beta-Lactam-Antibiotika: Ciprofloxacin plus Aminoglykosid oder Glykopeptid.

Bei Niereninsuffizienz: Pseudomonaswirksames Cephalosporin. biotikatherapie als Teil der supportiven Therapie empfohlen werden. Wer heute bei pädiatrisch-onkologischen Patienten mit Fieber bei Granulozytopenie und einem vermutlich niedrigen Risiko einer schweren Infektion die Therapie nach 24 Stunden intravenöser Behandlung auf ein orales Antibiotikum umstellt, kann sich nicht auf eine ausreichende wissenschaftliche Evidenz berufen. Dieser an sich viel versprechende Ansatz ist nur innerhalb korrekt geplanter, randomisierter, kontrollierter, multizentrischer und wenn möglich multinationaler Studien zu erproben [4].

Anmerkung: Eine prospektive multizentrische multinationale Studie (SPOG 2003 FN) ist derzeit initiiert. Die Studien sind offen für weitere Teilnehmer. Interessierte Zentren wenden sich bitte an Dr. R. Ammann (E-mail: roland.ammann@insel.ch).

\section{Literatur}

${ }^{1}$ Recommendations for preventing the spread of vancomycin resistance. Hospital Infection Control Practices Advisory Committee (HICPAC). Infect Control Hosp Epidemiol 1995; 16: 105-113

${ }^{2}$ Agaoglu L, Devecioglu O, Anak S et al. Cost-effectiveness of cefepime + netilmicin or ceftazidime + amikacin or meropenem monotherapy in febrile neutropenic children with malignancy in Turkey. J Chemother 2001; 13: $281-287$

${ }^{3}$ Ammann RA, Hirt A, Luthy AR et al. Predicting bacteremia in children with fever and chemotherapy-induced neutropenia. Pediatr Infect Dis J 2004; 23: 61 - 67

${ }^{4}$ Ammann RA, Simon A, de Bont ES. Low risk episodes of fever and neutropenia in pediatric oncology: Is outpatient oral antibiotic therapy the new gold standard of care? Pediatr Blood Cancer 2005; 45: 244 247

${ }^{5}$ Berger A, Kretzer V, Apfalter P et al. Safety evaluation of piperacillin/ tazobactam in very low birth weight infants. J Chemother 2004; 16: $166-171$

${ }^{6}$ Bodey GP, Rodriguez V, Chang HY et al. Fever and infection in leukemic patients: a study of 494 consecutive patients. Cancer 1978; 41 : $1610-1622$

${ }^{7}$ Chuang YY, Hung IJ, Yang CP et al. Cefepime versus ceftazidime as empiric monotherapy for fever and neutropenia in children with cancer. Pediatr Infect Dis J 2002; 21: 203 - 209

${ }^{8}$ Cometta A, Kern WV, De Bock R et al. Vancomycin versus placebo for treating persistent fever in patients with neutropenic cancer receiving piperacillin-tazobactam monotherapy. Clin Infect Dis 2003; 37: 382 389

${ }^{9}$ Cometta A, Zinner S, de Bock R et al. Piperacillin-tazobactam plus amikacin versus ceftazidime plus amikacin as empiric therapy for fever in granulocytopenic patients with cancer. The International Antimicrobial Therapy Cooperative Group of the European Organization for Research and Treatment of Cancer. Antimicrob Agents Chemother 1995; 39: $445-452$

${ }^{10}$ Creutzig U, Henze G, Bielack S et al. Krebserkrankung bei Kindern; Erfolg durch einheitliche Therapiekonzepte seit 25 Jahren. Dtsch Arztblatt 2003; 100 A: $842-852$

${ }^{11}$ Dompeling EC, Donnelly JP, Deresinski SC et al. Early identification of neutropenic patients at risk of grampositive bacteraemia and the impact of empirical administration of vancomycin. Eur J Cancer 1996; 32A: $1332-1339$

${ }^{12}$ Durack DT, Street AC. Fever of unknown origin - reexamined and redefined. Curr Clin Top Infect Dis 1991; 11: 35-51

${ }^{13}$ Elting LS, Rubenstein EB, Rolston K et al. Time to clinical response: an outcome of antibiotic therapy of febrile neutropenia with implications for quality and cost of care. J Clin Oncol 2000; 18: 3699-3706

${ }^{14}$ Elting LS, Rubenstein EB, Rolston KV et al. Outcomes of bacteremia in patients with cancer and neutropenia: observations from two decades of epidemiological and clinical trials. Clin Infect Dis 1997; 25: 247-259 
15 Engelhard D, Elishoov H, Strauss N et al. Nosocomial coagulase-negative staphylococcal infections in bone marrow transplantation recipients with central vein catheter. A 5-year prospective study. Transplantation 1996; 61: 430 - 434

16 Erjavec Z, de Vries-Hospers HG, Laseur M et al. A prospective, randomized, double-blinded, placebo-controlled trial of empirical teicoplanin in febrile neutropenia with persistent fever after imipenem monotherapy. J Antimicrob Chemother 2000; 45: 843 - 849

17 Feld R. Vancomycin as part of initial empirical antibiotic therapy for febrile neutropenia in patients with cancer: pros and cons. Clin Infect Dis 1999; 29: $503-507$

${ }^{18}$ Fleischhack G, Hartmann C, Simon A et al. Meropenem versus ceftazidime as empirical monotherapy in febrile neutropenia of paediatric patients with cancer. J Antimicrob Chemother 2001; 47: 841 - 853

19 Fleischhack G, Schmidt-Niemann M, Wulff B et al. Piperacillin, betalactam inhibitor plus gentamicin as empirical therapy of a sequential regimen in febrile neutropenia of pediatric cancer patients. Support Care Cancer 2001; 9: $372-379$

20 Flidel-Rimon O, Friedman S, Gradstein S et al. Reduction in multiresistant nosocomial infections in neonates following substitution of ceftazidime with piperacillin/tazobactam in empiric antibiotic therapy. Acta Paediatr 2003; 92: 1205-1207

${ }^{21}$ Freifeld A, Marchigiani D, Walsh T et al. A double-blind comparison of empirical oral and intravenous antibiotic therapy for low-risk febrile patients with neutropenia during cancer chemotherapy. N Engl J Med 1999; 341: $305-311$

22 Furno P, Bucaneve G, Del Favero A. Monotherapy or aminoglycosidecontaining combinations for empirical antibiotic treatment of febrile neutropenic patients: a meta-analysis. Lancet Infect Dis 2002; 2: $231-242$

23 Gobel V, Jurgens H, Thomas L et al. Microbial spectrum of blood and body cultures in febrile episodes of children under chemotherapy for treatment of malignant diseases. Pediatr Hematol Oncol 1987; 4: $7-13$

${ }^{24}$ Gorschluter M, Hahn C, Fixson A et al. Piperacillin-tazobactam is more effective than ceftriaxone plus gentamicin in febrile neutropenic patients with hematological malignancies: a randomized comparison. Support Care Cancer 2003; 11: 362 - 370

25 Graf N, Gobel U. Clinical trials and studies of the German Society for Paediatric Oncology and Haematology (GPOH) and the European Directive for the Implementation of Good Clinical Practice in the Conduct of Clinical Trials. Klin Padiatr 2004; 216: 129-131

${ }^{26}$ Groll AH, Lehrnbecher T. New antifungal drugs and the pediatric cancer patient: current status of clinical development. Klin Padiatr 2005; 217: 158 - 168

27 Hargrave DR, Hann II, Richards SM et al. Progressive reduction in treatment-related deaths in Medical Research Council childhood lymphoblastic leukaemia trials from 1980 to 1997 (UKALLVIII, X and XI). Br J Haematol 2001; 112: 293 - 299

28 Holdsworth M, Hanrahan J, Albanese B et al. Outpatient management of febrile neutropenia in children with cancer. Paediatr Drugs 2003; 5 : $443-455$

${ }^{29}$ Hughes WT, Armstrong D, Bodey GP et al. 2002 guidelines for the use of antimicrobial agents in neutropenic patients with cancer. Clin Infect Dis 2002; 34: 730-751

${ }^{30}$ Jacobs RF, Vats TS, Pappa KA et al. Ceftazidime versus ceftazidime plus tobramycin in febrile neutropenic children. Infection 1993; 21: $223-228$

31 Jones GR, Konsler GK, Dunaway RP et al. Risk factors for recurrent fever after the discontinuation of empiric antibiotic therapy for fever and neutropenia in pediatric patients with a malignancy or hematologic condition. J Pediatr 1994; 124 : $703-708$

32 Katz JA, Bash R, Rollins N et al. The yield of routine chest radiography in children with cancer hospitalized for fever and neutropenia. Cancer 1991; 68: 940-943

${ }^{33}$ Kebudi R, Gorgun O, Ayan I et al. Randomized comparison of cefepime versus ceftazidime monotherapy for fever and neutropenia in children with solid tumors. Med Pediatr Oncol 2001; 36: 434-441

${ }^{34}$ Klaassen RJ, Allen U, Doyle JJ. Randomized placebo-controlled trial of oral antibiotics in pediatric oncology patients at low-risk with fever and neutropenia [In Process Citation]. J Pediatr Hematol Oncol 2000; 22: $405-411$

35 Klastersky J, Paesmans M, Rubenstein EB et al. The Multinational Association for Supportive Care in Cancer risk index: A multinational scoring system for identifying low-risk febrile neutropenic cancer patients. J Clin Oncol 2000; 18: 3038 - 3051

${ }^{36}$ Kline RM, Baorto EP. Treatment of pediatric febrile neutropenia in the era of vancomycin-resistant microbes. Pediatr Blood Cancer 2005; 44: $207-214$

${ }^{37}$ Korones DN, Hussong MR, Gullace MA. Routine chest radiography of children with cancer hospitalized for fever and neutropenia: is it really necessary? Cancer 1997; 80: 1160 - 1164

38 Kresken M, Hafner D. Drug resistance among clinical isolates of frequently encountered bacterial species in central Europe during 1975-1995. Study Group Bacterial Resistance of the Paul-Ehrlich-Society for Chemotherapy. Infection 1999; 27 (Suppl 2): S2 - S 8

39 Lehrnbecher T, Stanescu A, Kuhl J. Short courses of intravenous empirical antibiotic treatment in selected febrile neutropenic children with cancer. Infection 2002; 30: $17-21$

${ }^{40}$ Lehrnbecher T, Varwig D, Kaiser J et al. Infectious complications in pediatric acute myeloid leukemia: analysis of the prospective multi-institutional clinical trial AML-BFM 93. Leukemia 2004; 18: $72-77$

${ }^{41}$ Lidsky K, Hoyen C, Salvator A et al. Antibiotic-resistant gram-negative organisms in pediatric chronic-care facilities. Clin Infect Dis 2002; 34 $760-766$

42 Link H, Blumenstengel K, Bohme A et al. [Antimicrobial therapy for fever of unknown origin in neutropenia. Standard recommendations of the Work Group of Infections in Hematology and Oncology of the German Association of Hematology and Oncology]. Dtsch Med Wochenschr 1999; 124 (Suppl 1): S3-S8

${ }^{43}$ Link H, Bohme A, Cornely OA et al. Antimicrobial therapy of unexplained fever in neutropenic patients-guidelines of the Infectious Diseases Working Party (AGIHO) of the German Society of Hematology and Oncology (DGHO), Study Group Interventional Therapy of Unexplained Fever, Arbeitsgemeinschaft Supportivmassnahmen in der Onkologie (ASO) of the Deutsche Krebsgesellschaft (DKG-German Cancer Society). Ann Hematol 2003; 82 (Suppl 2): S105-S117

44 Maschmeyer G, Link H, Hiddemann W et al. [Empirical antimicrobia therapy in neutropenic patients. Results of a multicenter study by the Infections in Hematology Study Group of the Paul Ehrlich Society]. Med Klin (Munich) 1994; 89: 114-123

45 Maschmeyer G, Link H, Hiddemann W et al. Pulmonary infiltrations in febrile patients with neutropenia. Risk factors and outcome under empirical antimicrobial therapy in a randomized multicenter study. Cancer 1994; 73: 2296-2304

46 Mullen CA. Ciprofloxacin in treatment of fever and neutropenia in pediatric cancer patients. Pediatr Infect Dis J 2003; 22: 1138 - 1142

47 Mullen CA, Petropoulos D, Roberts WM et al. Outpatient treatment of fever and neutropenia for low risk pediatric cancer patients. Cancer 1999; 86: $126-134$

${ }^{48}$ Nurnberger W, Bonig H, Burdach S et al. Tolerability of piperacillin/ tazobactam in children and adolescents after high dose radio-/chemotherapy and autologous stem cell transplantation. Infection 1998; 26 : 65- 67

49 Orudjev E, Lange BJ. Evolving concepts of management of febrile neutropenia in children with cancer. Med Pediatr Oncol 2002; 39: 77-85

50 Paganini H, Gomez S, Ruvinsky S et al. Outpatient, sequential, parenteral-oral antibiotic therapy for lower risk febrile neutropenia in children with malignant disease: a single-center, randomized, controlled trial in Argentina. Cancer 2003; 97: 1775 - 1780

51 Paganini HR, Sarkis CM, De Martino MG et al. Oral administration of cefixime to lower risk febrile neutropenic children with cancer. Cancer 2000; 88: $2848-2852$

52 Paul M, Benuri-Silbiger I, Soares-Weiser K et al. Beta lactam monotherapy versus beta lactam-aminoglycoside combination therapy for sepsis in immunocompetent patients: systematic review and metaanalysis of randomised trials. Bmj 2004; 328: 668

53 Paul M, Soares-Weiser K, Grozinsky S et al. Beta-lactam versus betalactam-aminoglycoside combination therapy in cancer patients with neutropaenia. Cochrane Database Syst Rev 2003: CD003038

54 Petrilli AS, Dantas LS, Campos MC et al. Oral ciprofloxacin vs. intravenous ceftriaxone administered in an outpatient setting for fever and neutropenia in low-risk pediatric oncology patients: randomized prospective trial. Med Pediatr Oncol 2000; 34: 87 - 91

55 Ramphal R, Bolger M, Oblon DJ et al. Vancomycin is not an essential component of the initial empiric treatment regimen for febrile neutropenic patients receiving ceftazadime: A randomized prospective study. Antimicro Agents Chemother 1992; 36: 1062 - 1067 
${ }^{56}$ Renoult E, Buteau C, Turgeon $\mathrm{N}$ et al. Is routine chest radiography necessary for the initial evaluation of fever in neutropenic children with cancer? Pediatr Blood Cancer 2004; 43: 224-228

57 Santolaya ME, Villarroel M, Avendano LF et al. Discontinuation of antimicrobial therapy for febrile, neutropenic children with cancer: a prospective study. Clin Infect Dis 1997; 25: $92-97$

58 Shenep JL, Flynn PM, Baker DK et al. Oral cefixime is similar to continued intravenous antibiotics in the empirical treatment of febrile neutropenic children with cancer. Clin Infect Dis 2001; 32: 36-43

${ }^{59}$ Silling G, Fegeler W, Roos N et al. Early empiric antifungal therapy of infections in neutropenic patients comparing fluconazole with amphotericin B/flucytosine. Mycoses 1999; 42 (Suppl 2): 101 - 104

60 Todeschini G, Franchini M, Tecchio C et al. Improved prognosis of Pseudomonas aeruginosa bacteremia in 127 consecutive neutropenic patients with hematologic malignancies. Int J Infect Dis 1998; 3: $99-104$

${ }^{61}$ Tunkel AR, Sepkowitz KA. Infections caused by viridans streptococci in patients with neutropenia. Clin Infect Dis 2002; 34: 1524-1529

62 Viscoli C, Castagnola E, Lint MT Van et al. Fluconazole versus amphotericin B as empirical antifungal therapy of unexplained fever in granulocytopenic cancer patients: a pragmatic, multicentre, prospective and randomised clinical trial. Eur J Cancer 1996; 32A: 814-820

${ }^{63}$ Wacker P, Halperin DS, Wyss M et al. Early hospital discharge of children with fever and neutropenia: a prospective study. J Pediatr Hematol Oncol 1997; 19: 208-211

${ }^{64}$ Wehl G, Allerberger F, Heitger A et al. Trends in infection morbidity in a pediatric oncology ward, 1986-1995. Med Pediatr Oncol 1999; 32: $336-343$ 